\title{
Effects of blood pressure level management on maternal and perinatal outcomes in pregnant women with mild to moderate gestational hypertension
}

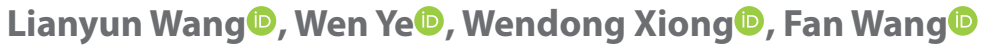 \\ Department of Obstetrics and Gynecology, The Second Affiliated Hospital and Yuying Children's Hospital \\ of Wenzhou Medical University, Wenzhou, Zhejiang, China
}

\begin{abstract}
Objectives: This study aims to investigate the effects of blood pressure control level on maternal and perinatal outcomes in pregnant women with mild to moderate gestational hypertension (GHp).

Material and methods: A total of 344 pregnant women who initially diagnosed as mild to moderate gestational hypertension were recruited in this study. They were divided into 4 groups according to the stabilized blood pressure level (BPL) during pregnancy. The clinical parameters and the incidence of adverse pregnancy outcomes were compared among the four groups. The association between blood pressure levels and relative factors were analyzed using the $x^{2}$ test. Multivariate logistic regression analysis was adopted for risk factors associated with adverse pregnancy outcomes.

Results: The results showed the prevalence of obesity was significantly associated with blood pressure levels of mild-moderate GHp pregnant women $(p=0.029)$. The incidence of severe GHp, SPE in group $A$, group $B$, and group $C$ were statistically significant ( $p<0.001, p=0.041$, respectively). In the patients who used drugs to control $B P L$, the incidence of severe GHp has a significant association with the initial blood pressure levels $(p=0.004)$. However, no significant difference was found in the incidence of $S P E, P E+$ Upro, and SGA (all $p>0.05$ ). Multivariate logistic regression analyses results showed that the gestational factor $\mathrm{BPL}$ was an independent risk factor for the incidence of $\mathrm{sGHp}$. The $\mathrm{AMA}$, primigravida, gestational $\mathrm{BPL}$, and edema were risk factors for the incidence of preeclampsia with proteinuria. To the incidence of SPE, gestational BPL is the independent risk factor. Finally, preeclampsia anamnesis and FGR trend are the high-risk parameters to the incidence of SGA.

Conclusions: Timely management and control of blood pressure in pregnant women with mild to moderate GHp were beneficial to reduce the occurrence of severe GHp and sPE, but the incidence of SGA does not affected.

Key words: blood pressure level; pregnancy; maternal outcome; perinatal outcome; preeclampsia; hypertension in pregnancy; adverse pregnant outcomes
\end{abstract}

Ginekologia Polska 2020; 91, 3: 137-143

\section{INTRODUCTION}

Hypertensive disorder complicating pregnancy (HDCP) is a common complication of pregnancy, with a spectrum of conditions that include chronic hypertension, gestational hypertension (GHp), preeclampsia (PE), severe PE (sPE), eclampsia, and chronic hypertension with preeclampsia. HDCP is one of the main causes of maternal and perinatal morbidity and mortality, which seriously affects the health of mother and fetal $[1,2]$. Pregnancy with hypertension affected $6-8 \%$ of pregnancies in the USA, and about $5.22 \%$ of pregnancies in China $[3,4]$. In addition to maternal cerebrovascular and cardiac complications, HDCP is also associated with small for gestational age (SGA) infants and preterm birth [5-7].
Previous study by Buchbinder A et al. [6] showed the adverse perinatal outcomes were higher in women with severe gestational hypertension than in mild preeclampsia. A study by Mudjari NS et al. [8] pointed that the management of hypertension in pregnancy by preventing women from getting the risks of increased blood pressure (BP) can reduce maternal and perinatal morbidity and mortality.

The prevention, early diagnosis, and treatment of HDCP can reduce the risk of maternal and fetal complications [9-11]. In most regions of the world, severe gestational hypertension was diagnosed when the blood pressure during pregnancy is more than $160 / 110 \mathrm{~mm} \mathrm{Hg}$ [12-14]. It has reached a consensus on the antihypertensive treatment 
of severe gestational hypertension, and it is necessary to control the blood pressure when the severe gestational hypertension occurs. However, there are still different views on when the treatment of hypertension should be initiated for pregnant women with mild-moderate gestational hypertension (systolic blood pressure 140-159 $\mathrm{mm} \mathrm{Hg}$ and diastolic blood pressure 90-109 $\mathrm{mm} \mathrm{Hg}$ ). According to the report of the American College of Obstetricians and Gynecologists' Task Force on hypertension in pregnancy, it is suggested that anti-hypertensive medications not be administered for women with mild gestational hypertension or preeclampsia with a persistent blood pressure of less than $160 / 110 \mathrm{~mm} \mathrm{Hg}$ [12]. Whereas some other countries, such as Canada, Australia, China, and so on, believe that the treatment of anti-hypertensive can also be considered in women with mild-moderate gestational hypertensive [13-15]. Therefore, to optimize the management of blood pressure in pregnancies with mild-moderate hypertensive is necessary for reducing maternal and perinatal morbidity and mortality.

\section{Objectives}

In the present study, we compared the clinical factors associated with GHp and incidence rates of severe GHp, preeclampsia with proteinuria (PE + Upro), sPE and SGA. This study aims to investigate the effects of blood pressure control level on perinatal outcomes in women with mild-moderate gestational hypertension.

\section{MATERIAL AND METHODS} Study Population

A total of 344 pregnant women (aged from 21 to 44 , the average age was $30.15 \pm 5.15$ years; gestational age of 20-32 weeks, the average gestational age was $25.75 \pm 2.24$ weeks) were included between January 2012 and December 2016 in The Second Affiliated Hospital and Yuying Children's Hospital of Wenzhou Medical University (Wenzhou, Zhejiang). Eligible women were those who have had regular antenatal examinations, carried the pregnancy to term, and initially diagnosed with mild to moderate gestational hypertensive. The women with multiple pregnancies, fetal chromosomal abnormalities, placenta previa, spontaneous abortion, or induced abortion before 20 weeks of pregnancy were excluded. The clinical information was collected in this study including maternal age, maternal pre-pregnancy $\mathrm{BMI}$, recurrent spontaneous abortion (RSA), previous history of preeclampsia, and maternal syndrome (such as nephritis and kidney disease syndrome, thyroid disorder, glucose metabolism, immune system disease, and polycystic ovarian syndrome). Obesity is usually classified by BMI. According to the criteria recommended by Working Group on Obesity in China, BMI was grouped into three categories: normal weight $\left(<24.0 \mathrm{~kg} / \mathrm{m}^{2}\right)$, over- weight ( $\geq 24.0$ and $<28 \mathrm{~kg} / \mathrm{m}^{2}$ ), and obesity $\left(\geq 28.0 \mathrm{~kg} / \mathrm{m}^{2}\right)$. The detailed clinical characteristics were summarized in Table 1 and Table 2. The study protocol was approved by the Ethical Approval of The Second Affiliated Hospital and Yuying Children's Hospital of Wenzhou Medical University. All participants signed informed consent.

\section{Definition and classification}

According to the stabilized blood pressure level (BPL) during pregnancy, the participants were divided into four groups, including $A, B, C$, and D group. Group $A$ includes 135 pregnancy women (BPL $<130 / 80 \mathrm{~mm} \mathrm{Hg}$ ). Group B includes 160 participants with systolic blood pressure between 130 and $139 \mathrm{~mm} \mathrm{Hg}$, and diastolic blood pressure between 80 and $89 \mathrm{~mm} \mathrm{Hg}$. Group C obtained 46 participants with systolic blood pressure between 140 and $149 \mathrm{~mm} \mathrm{Hg}$, and diastolic blood pressure between 90 and $99 \mathrm{~mm} \mathrm{Hg}$. Group D includes 3 participants with systolic blood pressure between 150 and $159 \mathrm{~mm} \mathrm{Hg}$, and diastolic blood pressure between 100 and $109 \mathrm{~mm} \mathrm{Hg}$.

\section{Diagnostic Criteria}

The diagnosis of HDCP, including GHp, PE, and SPE, was based on the diagnosis and treatment guideline of hypertensive disorders during pregnancy [16]. Pregnancy complications, obtaining gestational diabetes mellitus (GDM), small for gestational age (SGA), fetal growth restriction (FGR), polycystic ovarian syndrome (PCOS), immune system disease, and other gestational complications, were also analyzed in the study. The diagnosis of GDM was based on the results of an oral glucose tolerance test [17]. Wessel's modified criteria were used to define the SGA infants. The predictive factors of PE mainly include prehypertension, excess body mass (> $0.5 \mathrm{~kg}$ per week), edema, hypoalbuminemia, decreased platelet level, and FGR trend. PCOS was defined according to the consensus on women's health aspects of PCOS [18].

\section{Blood pressure control method}

The management of blood pressure during pregnancy was controlled using oral drug therapeutic approach (labetalol or nifedipine) and lifestyle interventions. Lifestyle interventions, such as rest, diet control, adjustment of mental and environmental factors, are of proven benefit in gestational hypertensive. Among the participants in this study, there are 48 pregnancy women used therapeutic drug approach to control the blood pressure. What's more, the placental and fetal growth status were detected during the anti-hypertensive treatment.

\section{Statistical analysis}

Statistical analysis was carried out using SPSS software (SPSS, Inc., Chicago, IL, USA). Data are expressed as 
Table 1. The relationship between blood pressure level and parameters of GHp women

\begin{tabular}{|c|c|c|c|c|c|c|}
\hline \multirow{2}{*}{ Parameters } & \multirow{2}{*}{ Cases $(n=344)$} & \multicolumn{4}{|c|}{ GHp women groups } & \multirow{2}{*}{$\mathbf{p}$} \\
\hline & & $A(n=135)$ & $B(n=160)$ & $C(n=46)$ & $D(n=3)$ & \\
\hline Gestational age & $25.75 \pm 2.24$ & $25.93 \pm 2.18$ & $25.46 \pm 2.18$ & $26.20 \pm 2.71$ & $26.33 \pm 3.51$ & 0.134 \\
\hline RSA & & & & & & 0.951 \\
\hline No & 323 & 126 & 151 & 43 & 3 & \\
\hline Yes & 21 & 9 & 9 & 3 & 0 & \\
\hline AMA & & & & & & 0.070 \\
\hline No & 254 & 100 & 125 & 27 & 2 & \\
\hline Yes & 90 & 35 & 35 & 19 & 1 & \\
\hline Obesity & & & & & & 0.029 \\
\hline No & 301 & 124 & 140 & 35 & 2 & \\
\hline Yes & 43 & 11 & 20 & 2 & 1 & \\
\hline PE anamnesis & & & & & & 0.182 \\
\hline No & 335 & 134 & 155 & 43 & 3 & \\
\hline Yes & 9 & 1 & 5 & 3 & 0 & \\
\hline Primigravida & & & & & & 0.056 \\
\hline No & 169 & 62 & 75 & 29 & 3 & \\
\hline Yes & 175 & 73 & 85 & 17 & 0 & \\
\hline Primiparity & & & & & & 0.524 \\
\hline No & 29 & 8 & 16 & 5 & 0 & \\
\hline Yes & 315 & 127 & 144 & 41 & 3 & \\
\hline Pregnancy complications & & & & & & 0.370 \\
\hline No & 204 & 82 & 95 & 24 & 3 & \\
\hline Yes & 140 & 53 & 65 & 22 & 0 & \\
\hline
\end{tabular}

RSA — recurrent spontaneous abortion; AMA — advanced maternal age (pregnant women aged 35 years or over)

\begin{tabular}{|c|c|c|c|c|c|c|}
\hline \multirow{2}{*}{ Outcomes } & \multirow{2}{*}{ Cases $(n=344)$} & \multicolumn{4}{|c|}{ GHp women groups } & \multirow{2}{*}{$\mathbf{p}$} \\
\hline & & $A(n=135)$ & $B(n=160)$ & $C(n=46)$ & $D(n=3)$ & \\
\hline Severe GHp & & & & & & $<0.001$ \\
\hline No & 322 & 134 & 149 & 36 & 3 & \\
\hline Yes & 22 & 1 & 11 & 10 & 0 & \\
\hline PE + Upro & & & & & & 0.311 \\
\hline No & 242 & 98 & 113 & 28 & 3 & \\
\hline Yes & 102 & 37 & 47 & 18 & 0 & \\
\hline sPE & & & & & & 0.041 \\
\hline No & 296 & 122 & 137 & 34 & 3 & \\
\hline Yes & 48 & 13 & 23 & 12 & 0 & \\
\hline SGA & & & & & & 0.813 \\
\hline No & 314 & 124 & 144 & 43 & 3 & \\
\hline Yes & 30 & 11 & 16 & 3 & 0 & \\
\hline
\end{tabular}

Severe GHp — severe gestational hypertension; PE + Upro — preeclampsia with proteinuria; sPE — severe preeclampsia; SGA — small for gestational age

mean \pm SD. The clinical profile and incidence of severe $\mathrm{GHp}$, preeclampsia with proteinuria ( $\mathrm{PE}+$ Upro), sPE, SGA were compared among the four groups. The differences between two groups were examined by paired student's t tests. Maternal and perinatal outcomes were compared using $x^{2}$ test and multivariable logistic regression to control for potential 
Table 3. Comparison of pregnancy blood pressure levels and prevalence of adverse pregnancy outcomes in $\mathbf{4 8}$ mild-moderate GHp women using anti-hypertensive drugs

\begin{tabular}{|c|c|c|c|c|c|}
\hline \multirow{2}{*}{ Outcomes } & \multirow{2}{*}{ Cases $(n=48)$} & \multicolumn{3}{|c|}{ Prenatal BPL (mm Hg) } & \multirow{2}{*}{$\mathbf{p}$} \\
\hline & & $<130 / 80$ & $(130-139) /(80-89)$ & $(140-149) /(90-99)$ & \\
\hline Severe GHp & & & & & 0.007 \\
\hline No & 31 & 9 & 18 & 4 & \\
\hline Yes & 17 & 1 & 7 & 9 & \\
\hline PE + Upro & & & & & 0.168 \\
\hline No & 24 & 7 & 13 & 4 & \\
\hline Yes & 24 & 3 & 12 & 9 & \\
\hline sPE & & & & & 0.042 \\
\hline No & 28 & 8 & 16 & 4 & \\
\hline Yes & 20 & 2 & 9 & 9 & \\
\hline SGA & & & & & 0.709 \\
\hline No & 39 & 9 & 20 & 10 & \\
\hline Yes & 9 & 1 & 5 & 3 & \\
\hline
\end{tabular}

risk factors. P value less than 0.05 was considered to be statistically significant.

\section{RESULTS}

\section{Association between blood pressure level and} clinical characteristics of pregnant women

In the present study, we compared the clinical characteristics of four groups of pregnant women with mild-moderate $\mathrm{GHp}$. The prevalence of obesity among pregnant women in group $A$ is $8.1 \%(11 / 135), 12.5 \%(20 / 160)$ in group $B$, $23.9 \%(11 / 46)$ in group $C$, and $33.3 \%(1 / 3)$ in group $D$, which increased following the rising of BPL. The results showed the prevalence of obesity was significantly associated with blood pressure levels of mild-moderate GHp pregnant women $(p=0.029$, Tab. 1$)$. However, no positive associations were found with other clinical characteristics, such as gestational age, RSA, advanced maternal age (AMA; pregnant women aged 35 years or over), PE anamnesis, primigravida, primiparity, pregnancy complications (all $p>0.05$, Tab. 1).

\section{Prevalence of adverse pregnancy outcomes in relation to blood pressure levels}

We also analyzed the incidence of adverse pregnancy outcomes in four groups of pregnant women. In group $D$, there was no occurrence of adverse pregnancy outcome. The incidence of severe GHp in group $A(0.7 \%, 1 / 135)$, group B $(6.9 \%, 11 / 160)$, and group C $(21.7 \%, 10 / 46)$ was statistically significant $(p<0.001, T a b .2)$. The incidence of $s P E$ was also found (a significant difference among group $\mathrm{A}$, group $\mathrm{B}$, and group $C)(p=0.041, T a b .2)$. However, there was no significant difference of incidence of preeclampsia with proteinuria (PE + Upro) and SGA (all p > 0.05).
The occurrence of adverse pregnancy outcomes in pregnant women with mild-moderate GHp after anti-hypertensive

In the present study, 48 participants were given anti-hypertensive drugs to control blood pressure. Among the pregnant women who used anti-hypertensive drugs, 10 pregnant women had BPL less than $130 / 80 \mathrm{~mm} \mathrm{Hg}$, 25 participants had a BPL of $(130-139) /(80-89) \mathrm{mm} \mathrm{Hg}$, and 13 pregnant women had a BPL of (140-149)/(90-99) mm $\mathrm{Hg}$. As shown in Table 3, the incidence rates of severe GHp and $s P E$ in pregnant women with different pregnancy blood pressure levels were significantly different (all $p<0.05$ ). Among different pregnancy blood pressure levels groups, there was no dramatical difference in the incidence of preeclampsia with proteinuria and SGA (all $p>0.05$ ).

As shown in Table 4, with the increase of initial blood pressure levels, the incidence of severe $\mathrm{GHp}(3 / 23,7 / 15,7 / 10)$ is significantly increased, which has a significant difference $(p=0.004)$. However, no significant difference was found in the incidence of $\mathrm{SPE}, \mathrm{PE}+$ Upro, and SGA (all p > 0.05).

\section{Risk parameters related to the incidence of adverse pregnancy outcomes}

To analyze the risk parameters correlated with adverse pregnancy outcomes, we used the logistic regression analysis. As shown in Table 5, the factors gestational BPL $(\mathrm{OR}=2.958,95 \% \mathrm{Cl}=1.293-6.766, \mathrm{p}=0.010)$ is the high risk parameters that significantly related to the incidence of sGHp. The AMA $(\mathrm{OR}=0.112,95 \% \mathrm{Cl}=0.047-0.265, \mathrm{p}<0.001)$, primigravida $(\mathrm{OR}=0.129,95 \% \mathrm{Cl}=0.070-0.238, \mathrm{p}<0.001)$, gestational $\mathrm{BPL}(\mathrm{OR}=1.903,95 \% \mathrm{Cl}=1.224-2.959, \mathrm{p}=0.004)$, and edema $(\mathrm{OR}=2.698,95 \% \mathrm{Cl}=1.360-5.351, \mathrm{p}=0.005)$ 
Table 4. Comparison of initial blood pressure levels and prevalence of adverse pregnancy outcomes in 48 mild-moderate GHp women using anti-hypertensive drugs

\begin{tabular}{|c|c|c|c|c|c|}
\hline \multirow{2}{*}{ Outcomes } & \multirow{2}{*}{ Cases $(n=48)$} & \multicolumn{3}{|l|}{ Initial BPL (mm Hg) } & \multirow{2}{*}{$\mathbf{p}$} \\
\hline & & $(140-149) /(90-99)$ & $(150-159) /(100-109)$ & $\geq 160 / 110$ & \\
\hline Severe GHp & & & & & 0.004 \\
\hline No & 31 & 20 & 8 & 3 & \\
\hline Yes & 17 & 3 & 7 & 7 & \\
\hline PE + Upro & & & & & 0.651 \\
\hline No & 24 & 13 & 7 & 4 & \\
\hline Yes & 24 & 7 & 8 & 6 & \\
\hline sPE & & & & & 0.392 \\
\hline No & 28 & 14 & 10 & 4 & \\
\hline Yes & 20 & 9 & 5 & 6 & \\
\hline SGA & & & & & 0.805 \\
\hline No & 39 & 18 & 13 & 8 & \\
\hline Yes & 9 & 5 & 2 & 2 & \\
\hline
\end{tabular}

Table 5. Logistic regression analysis of factors contributing to adverse pregnancy outcomes

\begin{tabular}{|l|l|l|l|l|}
\hline Outcomes & Variables & $\mathbf{p}$ & OR & $\mathbf{9 5 \%} \mathrm{Cl}$ \\
\hline sGHp & Gestational BPL & 0.010 & 2.958 & $1.293-6.766$ \\
\hline PE + Upro & AMA & $<0.001$ & 0.112 & $0.047-0.265$ \\
\hline & Primigravida & $<0.001$ & 0.129 & $0.070-0.238$ \\
\hline & Gestational BPL & 0.004 & 1.903 & $1.224-2.959$ \\
\hline & Edema & 0.005 & 2.698 & $1.360-5.351$ \\
\hline SPE & Gestational BPL & 0.030 & 1.814 & $1.060-3.104$ \\
\hline SGA & PE anamnesis & 0.020 & 6.866 & $1.347-34.998$ \\
\hline & FGR trend & 0.004 & 3.993 & $1.565-10.189$ \\
\hline
\end{tabular}

FGR trend - uterine length and abdominal circumference were all below the 10th percentile for 3 consecutive weeks

are risk factors for the incidence of preeclampsia with proteinuria. To the incidence of $s P E$, gestational $B P L(O R=1.814$, $95 \% \mathrm{Cl}=1.060-3.104, \mathrm{p}=0.030)$ is the independent risk factor. Finally, preeclampsia anamnesis $(\mathrm{OR}=6.866$, $95 \% \mathrm{Cl}=1.347-34.998, \mathrm{p}=0.021)$ and $\mathrm{FGR}$ trend $(\mathrm{OR}=3.993$, $95 \% \mathrm{Cl}=1.565-10.189, \mathrm{p}=0.004)$ are the high risk parameters that dramatically associated with the incidence of SGA.

\section{DISCUSSION}

Gestational hypertensive is one of the common complications in pregnancy, which is initial occurred hypertension after 20 weeks of gestation and will return to normal within 12 weeks after delivery. Light GHp can be asymptomatic or mild dizziness, slightly elevated blood pressure, accompanied by edema or mild proteinuria, severe GHp may cause important organs injury, occur PE or eclampsia. PE and eclampsia are the main cause of adverse maternal and perinatal outcomes, such as intrauterine growth restriction and preterm birth [19, 20]. The study by Schokker SA et al. [21] showed previous hypertensive disorders of pregnancy was an independent risk factor for later vascular morbidity. Therefore, controlling pregnancy blood pressure can avoid organ or placenta injury and reduce the occurrence of serious adverse maternal and perinatal outcomes.

Previous studies have shown that the treatment of severe $\mathrm{GHp}$ can minimize the fluctuation of blood pressure during pregnancy and reduce the future risk of vascular (such as cerebrovascular and/or cardiovascular disease) disease [21-25]. A study by Choi DJ et al. [22] showed that a family history of premature cardiovascular disease was significantly associated with gestational hypertensive disease. The study by Abalos E et al. [23] showed that with the use of antihypertensive drugs could reduce the risk of developing severe hypertension, but no clear differences in the risk of other developing outcomes were found. Molvi SN et al. [24] also found antihypertensive therapy was associated with a lower incidence of severe pregnancy-induced hypertension, proteinuria, SGA babies, as well as some other maternal and fetal-neonatal non-fatal adverse events. A previous study suggested that anti-hypertensive treatment when $\mathrm{BPL}>140 / 90 \mathrm{~mm} \mathrm{Hg}$, coupled with close fetal monitoring, might result in both improved fetal outcome, as well as decreasing immediate maternal complications and permanent vascular injury [25]. However, whether anti-hypertensive drug therapy for women with mild or moderate GHp is still controversial.

In the present study, we analyzed the clinical parameters of pregnant women with mild to moderate GHp. We found the prevalence of obesity was significantly associated with blood pressure levels of mild-moderate GHp pregnant women. Some studies have indicated obesity was associated 
with blood pressure and increased risks of GHp. For instance, Gaillard R et al. [26] suggested maternal obesity and morbid obesity were strongly associated with the risk of gestational hypertensive disorders. Pregnancy is a period of substantial change in blood pressure, with physiological blood pressure decreasing before the middle stage of pregnancy, and then increasing until delivery [27-29]. In this study, we also analyzed the incidence of adverse pregnancy outcomes in pregnant women with different BPL. The results showed that the incidence of severe GHp in group $A(0.7 \%, 1 / 135)$, group $B(6.9 \%, 11 / 160)$, and group C $(21.7 \%, 10 / 46)$ was statistically significant, which showed an increasing trend with the BPL level. The incidence of sPE was consistent with that of severe GHp. But in group D, there was no occurrence of adverse pregnancy outcome, which might be related to only 3 cases, as the small sample size was prone to bias. Then we further investigated the adverse pregnancy outcomes in pregnant women who took BPL medications. Among 48 pregnant women who used anti-hypertensive drugs, 10 pregnant women had BPL less than 130/80 mm Hg, 25 participants had a BPL of (130-139)/(80-89) $\mathrm{mm} \mathrm{Hg}$, and 13 pregnant women had a BPL of (140-149)/(90-99) mm Hg. After anti-hypertensive treatment, the incidence rates of severe $\mathrm{GHp}$ and severe eclampsia in pregnant women with different pregnancy blood pressure levels were also found significantly different. However, there was no dramatical difference in the incidence of preeclampsia with proteinuria and SGA. The occurrence and development of mild to moderate GHp may be influenced by multiple factors. Multivariate logistic regression analysis results showed gestational BPL was an independent risk factor for the incidence of severe $\mathrm{GHp}$ and sPE. Hence, low blood pressure during pregnancy in $\mathrm{GHp}$ women can help reduce the occurrence of severe GHp and $\mathrm{SPE}$, and will not lead to increased incidence of SGA.

What's more, we analyzed initial blood pressure levels and prevalence of adverse pregnancy outcomes in mild-moderate $\mathrm{GHp}$ women using anti-hypertensive drugs. The results showed that the incidence of preeclampsia with proteinuria, SPE, and SGA have no significant difference with the initial BPL in mild to moderate pregnant women who had anti-hypertensive treatment, and only the difference in the incidence of severe GHp was statistically significant. The results indicated with the increase of initial blood pressure levels, the incidence of severe GHp (3/23, $7 / 15,7 / 10)$ was significantly increased. According to the multivariate logistic regression analysis results, the initial BPL was an independent risk factor for the incidence of severe $\mathrm{GHp}$, which suggested timely management and control of blood pressure in pregnant women with mild to moderate $\mathrm{GHp}$ was beneficial to reduce the occurrence of severe $\mathrm{GHp}$.

The multivariate logistic regression analysis results also showed edema was risk factor that significantly associated with preeclampsia with proteinuria. The pregnant women with edema show a higher incidence of preeclampsia with proteinuria than those without edema. Therefore, it is necessary to observe the occurrence of edema for preventing the incidence of PE. According to the results of detection of placental and fetal growth status during the anti-hypertensive treatment, there was no affection to the incidence of SGA. Multivariate logistic regression analysis results show the factors gestational BPL is the high-risk parameters that significantly related to the incidence of sGHp. The AMA, primigravida, gestational $B P L$, and edema are risk factors for the incidence of preeclampsia with proteinuria. To the incidence of $S P E$, gestational BPL is an independent risk factor. Finally, preeclampsia anamnesis and FGR trend are the high-risk parameters that dramatically associated with the incidence of SGA. These results showed that the risk factors influencing the incidence of adverse pregnancy outcomes were very complicated. The gestational BPL was found significantly associated with the incidence of severe GHp, preeclampsia with proteinuria, and sPE. To control the gestational BPL is beneficial to delay the progression of severe GHp, and reduce the incidence of sPE.

In considering the results of this study, some limitations need to be addressed. First, the sample size of patients is limited. A large number of subjects can improve the accuracy of the results. Second, part of the clinical data was not completed, and more parameters can be involved in the further analyses. Due to the limitations, further analyses are necessary for large research cohort and more parameters.

\section{CONCLUSIONS}

In conclusion, timely management and control of blood pressure in pregnant women with mild to moderate GHp was beneficial to delay the progression of severe GHp, and reduce the occurrence of SPE. What's more, there is no correlation was found with the incidence of SGA. Meanwhile, due to the regular prenatal examination and the diversification of diagnostic methods, mild to moderate GHp pregnant women can be timely diagnosed.

\section{Funding}

This study was funded by National Youth Natural Science Foundation of China (81501280), Natural Science Foundation of Zhejiang Provincial (LY15H040012), and Zhejiang Science and Technology Health Department Research Fund Project (2018260097).

\section{REFERENCES}

1. Berg C, Callaghan W, Henderson Z, et al. Pregnancy-Related Mortality in the United States, 1998 to 2005. Obstet Gynecol. 2011; 117(5): 1230 doi: 10.1097/aog.0b013e31821769ed.

2. Sibai B, Dekker G, Kupferminc M. Pre-eclampsia. Lancet. 2005; 365(9461): 785-799, doi: 10.1016/S0140-6736(05)17987-2, indexed in Pubmed: 15733721. 
3. Report of the National High Blood Pressure Education Program Working Group on High Blood Pressure in Pregnancy. Am J Obstet Gynecol. 2000; 183(1): s1-s22, doi: 10.1067/mob.2000.107928.

4. Ye C, Ruan Y, Zou L, et al. The 2011 survey on hypertensive disorders of pregnancy (HDP) in China: prevalence, risk factors, complications, pregnancy and perinatal outcomes. PLoS One. 2014; 9(6): e100180, doi: 10.1371/journal.pone.0100180, indexed in Pubmed: 24937406.

5. Sibai BM, Gordon T, Thom E, et al. Risk factors for preeclampsia in healthy nulliparous women: a prospective multicenter study. The National Institute of Child Health and Human Development Network of Maternal-Fetal Medicine Units. Am J Obstet Gynecol. 1995; 172(2 Pt 1): 642-648, doi: 10.1016/0002-9378(95)90586-3, indexed in Pubmed: 7856699.

6. Buchbinder A, Sibai BM, Caritis S, et al. National Institute of Child Health and Human Development Network of Maternal-Fetal Medicine Units. Adverse perinatal outcomes are significantly higher in severe gestational hypertension than in mild preeclampsia. Am J Obstet Gynecol. 2002; 186(1): 66-71, doi: 10.1067/mob.2002.120080, indexed in Pubmed: 11810087.

7. Ankumah NA, Cantu J, Jauk V, et al. Risk of adverse pregnancy outcomes in women with mild chronic hypertension before 20 weeks of gestation. Obstet Gynecol. 2014; 123(5): 966-972, doi: 10.1097/AOG.0000000000000205, indexed in Pubmed: 24785847.

8. Mudjari NS, Samsu N. Management of hypertension in pregnancy. Acta Med Indones. 2015; 47(1): 78-86, indexed in Pubmed: 25948773.

9. Collier AC, Sato BLM, Milam KA, et al. Methamphetamine, smoking, and gestational hypertension affect norepinephrine levels in umbilical cord tissues. Clin Exp Obstet Gynecol. 2015; 42(5): 580-585, indexed in Pubmed: 26524802.

10. Zhou DX, Bian XY, Cheng XY, et al. Late gestational liver dysfunction and its impact on pregnancy outcomes. Clin Exp Obstet Gynecol. 2016; 43(3): 417-421, indexed in Pubmed: 27328504.

11. Chen YS, Shen L, Mai RQ, et al. Levels of microRNA-181b and plasminogen activator inhibitor-1 are associated with hypertensive disorders complicating pregnancy. Exp Ther Med. 2014; 8(5): 1523-1527, doi: 10.3892/etm.2014.1946, indexed in Pubmed: 25289053.

12. American College of Obstetricians and Gynecologists, Task Force on Hypertension in Pregnancy. Hypertension in pregnancy. Report of the American College of Obstetricians and Gynecologists'Task Force on Hypertension in Pregnancy. Obstet Gynecol. 2013; 122(5): 1122-1131, doi: 10.1097/01.AOG.0000437382.03963.88, indexed in Pubmed: 24150027.

13. Magee LA, Pels A, Helewa M, et al. SOGC Hypertension Guideline Committee, Canadian Hypertensive Disorders of Pregnancy Working Group, Canadian Hypertensive Disorders of Pregnancy (HDP) Working Group. Diagnosis, evaluation, and management of the hypertensive disorders of pregnancy. Pregnancy Hypertens. 2014; 4(2): 105-145, doi: 10.1016/j. preghy.2014.01.003, indexed in Pubmed: 26104418.

14. Pfaff NF. The new hypertensive guidelines for pregnancy: what every nurse should know. J Perinat Neonatal Nurs. 2014; 28(2): 91-93, doi: 10.1097/JPN.0000000000000028, indexed in Pubmed: 24781764.

15. Kildea S, Gao Yu, Rolfe M, et al. Remote links: Redesigning maternity care for Aboriginal women from remote communities in Northern Australia A comparative cohort study. Midwifery. 2016; 34: 47-57, doi: 10.1016/j. midw.2016.01.009, indexed in Pubmed: 26971448.

16. Chinese Medical Association, Hypertensive Disorders in Pregnancy Subgroup Chinese Society of Obstetrics and Gynecology Chinese Medical Association. Diagnosis and treatment guideline of hypertensive disorders in pregnancy. Zhonghua Fu Chan Ke Za Zhi. 2015, doi: 10.1016/c2012-0-02662-2.
17. American Diabetes Association. Executive summary: Standards of medical care in diabetes--2012. Diabetes Care. 2012; 35 Suppl 1: S4-SS10, doi: 10.2337/dc12-s004, indexed in Pubmed: 22187471.

18. Fauser BC, Tarlatzis BC, Rebar RW, et al. Consensus on women's health aspects of polycystic ovary syndrome (PCOS): the Amsterdam ESHRE/ASRM-Sponsored 3rd PCOS Consensus Workshop Group. Fertil Steril. 2012; 97(1): 28-38.e25, doi: 10.1016/j.fertnstert.2011.09.024, indexed in Pubmed: 22153789.

19. Abalos E, Cuesta C, Carroli G, et al. WHO Multicountry Survey on Maternal and Newborn Health Research Network. Pre-eclampsia, eclampsia and adverse maternal and perinatal outcomes: a secondary analysis of the World Health Organization Multicountry Survey on Maternal and Newborn Health. BJOG. 2014; 121 Suppl 1: 14-24, doi: 10.1111/14710528.12629, indexed in Pubmed: 24641531.

20. Madazli R, Yuksel MA, Imamoglu M, et al. Comparison of clinical and perinatal outcomes in early- and late-onset preeclampsia. Arch Gynecol Obstet. 2014; 290(1): 53-57, doi: 10.1007/s00404-014-3176-x, indexed in Pubmed: 24549271.

21. Schokker SAM, Van Oostwaard MF, Melman EM, et al. Cerebrovascular, cardiovascular and renal hypertensive disease after hypertensive disorders of pregnancy. Pregnancy Hypertens. 2015; 5(4): 287-293, doi: 10.1016/j.preghy.2015.06.002, indexed in Pubmed: 26597742.

22. Choi DJ, Yoon $\mathrm{CH}_{\text {, Lee }} \mathrm{H}$, et al. The Association of Family History of Premature Cardiovascular Disease or Diabetes Mellitus on the Occurrence of Gestational Hypertensive Disease and Diabetes. PLoS One. 2016; 11(12): e0167528, doi: 10.1371/journal.pone.0167528, indexed in Pubmed: 27918585.

23. Abalos E, Duley L, Steyn DW, et al. Antihypertensive drug therapy for mild to moderate hypertension during pregnancy. Cochrane Database Syst Rev. 2001; 10(2): CD002252, doi: 10.1002/14651858.CD002252, indexed in Pubmed: 11406040.

24. Molvi SN, Mir S, Rana VS, et al. Role of antihypertensive therapy in mild to moderate pregnancy-induced hypertension: a prospective randomized study comparing labetalol with alpha methyldopa. Arch Gynecol Obstet. 2012; 285(6): 1553-1562, doi: 10.1007/s00404-011-2205-2, indexed in Pubmed: 22249781.

25. Scantlebury DC, Schwartz GL, Acquah LA, et al. The treatment of hypertension during pregnancy: when should blood pressure medications be started? Curr Cardiol Rep. 2013; 15(11): 412, doi: 10.1007/s11886-0130412-0, indexed in Pubmed: 24057769.

26. Gaillard R, Bakker R, Steegers EAP, et al. Associations of maternal obesity with blood pressure and the risks of gestational hypertensive disorders. The Generation R Study. J Hypertens. 2011; 29(5): 937-944, doi: 10.1097/HJH.0b013e328345500c, indexed in Pubmed: 21430559.

27. Macdonald-Wallis C, Tilling K, Fraser A, et al. Associations of blood pressure change in pregnancy with fetal growth and gestational age at delivery: findings from a prospective cohort. Hypertension. 2014; 64(1): 36-44, doi: 10.1161/HYPERTENSIONAHA.113.02766, indexed in Pubmed: 24821945

28. Macdonald-Wallis C, Lawlor DA, Fraser A, et al. Blood pressure change in normotensive, gestational hypertensive, preeclamptic, and essential hypertensive pregnancies. Hypertension. 2012; 59(6): 1241-1248, doi: 10.1161/HYPERTENSIONAHA.111.187039, indexed in Pubmed: 22526257.

29. Chen Z, Liu W, Sun X, et al. Clinical study on the association between pregnancy-induced hypertension and insulin resistance. Exp Ther Med. 2017; 13(5): 2065-2070, doi: 10.3892/etm.2017.4169, indexed in Pubmed: 28565809 . 